Math. Model. Nat. Phenom.

Vol. 3, No. 1, 2008, pp. 75-97

\title{
The Effect of Crystal-Melt Surface Energy on the Stability of Ultra-Thin Melt Films
}

\author{
M. Beerman ${ }^{a}$ and L. N. Brush ${ }^{b 1}$ \\ a Andrews Space Inc., Seattle, WA, 98104, USA \\ ${ }^{b}$ Department of Materials Science and Engineering, \\ University of Washington, Seattle, WA, 98195, USA
}

\begin{abstract}
The stability and evolution of very thin, single component, metallic-melt films is studied by analysis of coupled strongly nonlinear equations for gas-melt (GM) and crystalmelt (CM) interfaces, derived using the lubrication approximation. The crystal-melt interface is deformable by freezing and melting, and there is a thermal gradient applied across the film. Linear analysis reveals that there is a maximum applied far-field temperature in the gas, beyond which there is no film instability. Instabilities observed in the absence of CM surface energy are oscillatory for all marginally stable states. The effect of the CM surface energy is to expand the parameter range over which a film is unstable. The new range of instabilities are of longer wavelength and are stationary, compared to the range found in the absence of CM surface energy. Numerical analysis illustrates how perturbations grow to rupture by standing waves. With CM surface energy, an initially longer (stationary) wavelength perturbation has a relatively slow growth rate, but it can trigger the appearance of much faster growing shorter wavelength (oscillatory) instabilities, leading to an accelerated film rupture process.
\end{abstract}

Key words: phase change, lubrication theory, interface instability, nonlinear, latent heat, crystal, van der Waals, long wavelength

AMS subject classification: 76D08, 76E17, 76E30, 80A22

\footnotetext{
${ }^{1}$ Corresponding author. Email: brush@u.washington.edu
} 


\section{Introduction}

The onset of morphological instability and the subsequent evolution of thin liquid films is important in a variety of applications such as the processing of foams, laser micromachining and the study of liquid multilayers. Often the micro- and nanoscale phenomena occurring in these applications determines the resulting bulk scale behavior and properties of the systems. The films may also be undergoing phase changes such as solidification, which gives rise to additional physical effects.

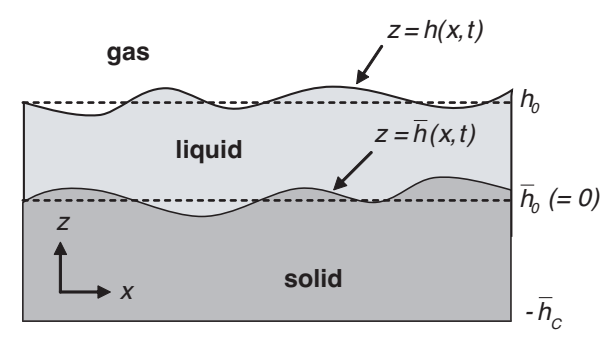

Figure 1: A two dimensional thin melt film is bounded by crystal-melt $\bar{h}(x, t)$ and melt-gas $h(x, t)$ interfaces. Dashed lines $h_{0}$ and $\bar{h}_{0}$ represent a uniformly thick layer which is used as a base state in a linear stability analysis. The crystal is a passive thin layer. A linear temperature gradient is applied in the transverse direction at $z=-\bar{h}_{C}$. Longitudinal $x$ and transverse $z$ directions are indicated in the figure. (Modified from [1]).

This work was motivated by considering transverse solidification of thin melt films as one of many freezing geometeries that are present in bulk metallic foam processing. However, the results can also apply to a variety of thin-film systems. The problem is unique since it involves local solidification/melting and is an application of long-wave/lubrication theory in a system having two interfaces which are deformable but which are different in nature. Such thin films lie at the boundary between continuum mechanics and mesoscale dynamics, where intermolecular forces compete with the collective behavior of molecular systems giving rise to unique phenomena.

In a previous study, the authors have derived a new model for the evolution of a thin melt film bounded by a gas-liquid interface and a crystal-melt interface deformable by freezing or melting [1]. The crystal and melt were assumed to be a single component system, i.e. there were no concentration effects due to the presence of an alloying species. An applied thermal gradient was included, oriented in a direction transverse to the film and this research focused on instabilities that ultimately lead to rupture. Results showed that compared to the case of an isothermal, thin-liquid film resting on a substrate, in which a band of stationary modes is unstable, the applied temperature gradient and the crystal-melt interface change the marginal stability condition in two ways. First, the applied gradient stabilizes the system, in other words, there is an upper bound on its magnitude beyond which instabilities are entirely suppressed. Secondly, the onset of instability is an oscillatory Hopf bifurcation. Numerical 
results were carried out showing how oscillatory instabilities can lead to rupture by standing waves.

However, in this previous work, the effect of the crystal-melt surface energy was neglected so that the crystal-melt interface was assumed to be an isotherm. This article focuses on the effect of the (isotropic) crystal-melt surface energy on instability and evolution of a thin melt film. The effect of the crystal-melt surface energy is to render an unstable interface non-isothermal, as given by the Gibbs-Thomson condition. This effect can fundamentally alter the stability landscape and the nonlinear evolution of an unstable thin melt film as compared to the results of the previous work.

It is noted here that this analysis treats those ranges of crystal-melt interface orientations which do not exhibit surface premelting. Premelting is when a thin film of a melt appears between a crystal and a vapor near the bulk melting point of the material[2] [3]. (See the recent article [4] and references therein.) In metals and alloys non-premelting interfaces are included in those ranges of crystallographic orientations surrounding close-packed orientations [5].

The paper continues below with a brief background section, followed by a listing of the governing dimensional differential equations in the bulk, at the interfaces and in the farfield. In this work lubrication theory is applied to treat the thin film, and so the scalings are introduced and then the non-dimensional evolution equations for the two interfaces are presented. The details of the derivations are omitted because they are the same as given in Beerman and Brush[1]. Results of the linear theory are then presented, numerical solutions are shown and a discussion follows.

\section{Background}

One example of thin films undergoing solidification arises in the processing a metallic foam network [6] [7], in which there are lamella separated by Plateau borders. Solid metal foams are formed by cooling a molten metal mixed with gas in such a way that the gas bubbles, it is hoped, are preserved inside the material after solidification. In order to successfully process a foam network, the liquid in the thin film regions must solidify before rupture occurs. Rupture occurs by means of the evolution of an instability of a thinning liquid lamella and in metals this typically occurs in milliseconds. Rupture results in coarsening of the foam network. Since gas - liquid metal foams are highly unstable, particulate foaming agents such as $\mathrm{Al}_{2} \mathrm{O}_{3}, \mathrm{SiC}$ or $\mathrm{MgO}$ are required [7], which increases the effective viscosity in order to reduce drainage and bubble coalescence rates [8]. These fine particles may have deleterious effects on the resulting material properties of the solid foam. Breward [9] provides an overview of evolution and stability of liquid foam networks, and Brush and Davis [10] have derived an alternative thinning law for a foam lamella. The question of how to cleanly freeze a metallic foam remains open.

Uni-directional solidification of foam can occur as it passes through a temperature gradient. In a foam with thousands of bubbles, the lamella may have any orientation with 
respect to the applied gradient. In order to enable a clear understanding of the behavior of the lamella during freezing we investigate the case of transverse freezing, where the solidification front moves normal to the liquid film boundaries. Other freezing geometries may result in triple junctions where all three phases (passive gas, melt and crystal) meet, making it difficult to interpret the results without an understanding of the solidification of a film in the absence of triple junctions. This analysis represents the first steps toward developing an understanding of the effects of solidification and an applied thermal gradient on film rupture in foam.

Besides foams, another application involving a transverse heat flow and solidification configuration in a thin film is pulsed laser melting, which is a processing technique used in the semiconductor industry for etching microcircuit elements, and for micro- and nanofluidics [11]. In this technique, a tightly focused laser is used to irradiate a surface with a Gaussian distribution of high energy photons. A thin disk region of material is liquefied, producing a film bounded on one side by its crystal and on the other by a melt-gas interface. One significant difference is that a longitudinal temperature gradient develops due to the Gaussian energy intensity distribution of the laser flux [12]. Hence, Marangoni effects will heavily influence the interface morphology. Models for this configuration have also incorporated evaporative mass transfer effects due to the high-energy flux. The thin film approximation is still applicable, and a non-linear equation for the film thickness has been derived [13]. Experimental work has shown that the interface can undergo instabilities that lead to droplet and ring formations that may be exploited for circuit fabrication [14].

Since there has been a great deal of analysis of the instability and evolution of thin films, (see the review article by [15]) and since Beerman and Brush[1] provide a review of much of the pertinent literature related to this particular problem, here we will briefly mention only theoretical work which has a direct bearing on this research.

The study of Williams \& Davis [16] serves as a benchmark for the work presented here. These authors carried out the linear stability analysis of an isothermal liquid-gas interface of a thin film on a horizontal substrate in the lubrication limit. The interface is unstable to a band of wavenumbers. They also carried out numerical solutions of the nonlinear evolution equation and showed how nonlinear effects accelerate growth of the instability.

There have also been studies of the evolution of non-isothermal films on substrates in which there are phase transitions at an interface. For example, Burelbach et al [17] utilized long-wave theory to derive evolution equations for gas-liquid interfaces during evaporation and condensation. Recently, Grigoriev [18] recommended a thermal control procedure to stabilize a thin film during drying.

The behavior of multilayered films, involving more than one interface have also been analyzed. These efforts are somewhat similar to the work presented here in that there are two deformable interfaces. For example, Bandyopadhyay et al. studied the stability and the evolution of interfaces separating multiple thin layers[19] and Fisher and Golovin [20] have analyzed a system of strongly non-linear evolution equations for the two gas-liquid interfaces bounding a two-layer liquid system. The latter analysis revealed oscillatory instabilities. In these examples interfaces were gas-liquid, liquid-liquid and/or liquid-substrate, but not 
crystal-melt.

\section{The Model}

We consider a thin film with a gradient applied in the transverse direction as shown in Fig. 1. In this paper the analysis is for the two-dimensional case. Therefore, the results apply to sections of films in which there is no interfacial curvature in the direction out of the page (normal to Fig. 1). The instabilities uncovered in this work correspond then to rolls extending out of the page. In a fully three-dimensional treatment these 'rolls' extending out of the page may compete with interface instabilities having different symmetry. Nevertheless, although there may be other patterns formed in three dimensions, the mechanisms giving rise to the instabilities shown here should remain relevant in three dimensions.

The longitudinal and transverse directions of the film are aligned with the $x$ and $z$ coordinates, respectively. In two dimensions, the procedure used to derive evolution equations for the CM interface $(z=\bar{h}(x, t))$ and for the MG interface $(z=h(x, t))$, is given in Beerman and Brush [1]. (Unperturbed upper $\left(h_{0}\right)$ and lower $\left(\bar{h}_{0}\right)$ surfaces are represented by dashed lines.) Although all results are presented in dimensionless form, all variables and parameters used to generate the plots and figures are taken from data for $\mathrm{Al}$, which is included in Tables of previous work [1]

The components of fluid velocity $(u(x, z, t), w(x, z, t))$, pressure $(p(x, z, t))$ and temperature $(T(x, z, t))$ are governed by the Navier-Stokes equations, the continuity equation and the heat transport equation,

$$
\begin{gathered}
\rho^{l}\left(u_{t}+u u_{x}+w u_{z}\right)=-(p+\phi)_{x}+\mu\left(u_{x x}+u_{z z}\right), \\
\rho^{l}\left(w_{t}+u w_{x}+w w_{z}\right)=-(p+\phi)_{z}+\mu\left(w_{x x}+w_{z z}\right), \\
u_{x}+w_{z}=0 \\
\rho^{l} c^{l}\left(T_{t}^{l}+u T_{x}^{l}+w T_{z}^{l}\right)=k^{l}\left(T_{x x}^{l}+T_{z z}^{l}\right),
\end{gathered}
$$

respectively, where the subscripts $t, x$ and $z$ represent partial derivatives. $\phi$ is a body force potential, for example, long range van der Waals molecular forces, $\rho^{l}$ is the density of the fluid and $\mu$ is the liquid viscosity. Temperature in the crystal $T^{s}$ is governed by

$$
\rho^{s} c^{s} T_{t}^{s}=k^{s}\left(T_{x x}^{s}+T_{z z}^{s}\right),
$$

where $c^{l}\left(c^{s}\right)$ is the heat capacity of the liquid (solid), $k^{l}\left(k^{s}\right)$ is the thermal conductivity of the liquid (solid) and $\rho^{s}$ is the density of the crystal.

At the MG interface $z=h(x, t)$, the following conditions apply:

$$
\begin{gathered}
w=h_{t}+u h_{x}, \\
-p+p_{G}+\frac{2 \mu}{\left(1+h_{x}^{2}\right)}\left[\left(u_{x} h_{x}^{2}+w_{z}\right)-h_{x}\left(u_{z}+w_{x}\right)\right]=\gamma^{M G} \frac{h_{x x}}{\left(1+h_{x}^{2}\right)^{3 / 2}},
\end{gathered}
$$




$$
-2 \mu\left(u_{x}-w_{z}\right) h_{x}+\mu\left(u_{z}+w_{x}\right)\left(1-h_{x}^{2}\right)=\gamma_{x}^{M G}\left(1+h_{x}^{2}\right)^{1 / 2} .
$$

and

$$
-k^{l}\left(\nabla T^{l} \cdot \hat{n}\right)=\alpha_{T h}\left(T^{l}-T_{\infty}\right) .
$$

These equations are the interface kinematic condition, jump conditions on the normal and the tangential components of the shear stress and the heat transfer condition at the MG interface, respectively. The gas phase is assumed inviscid. The unit normal vector at the MG interface is

$$
\hat{n}=\frac{-h_{x} \hat{i}+\hat{k}}{\left(1+h_{x}^{2}\right)^{1 / 2}},
$$

pointing from the liquid into the gas phase. In the above, the components of the fluid velocity and the temperature are all evaluated at the interface. $p_{G}$ is the hydrostatic pressure in the gas and $\alpha_{T h}$ is the heat transfer coefficient. The MG surface energy is assumed to depend linearly on the temperature so that

$$
\gamma^{M G}\left(T^{l}\right)=\gamma_{0}^{M G}-\gamma_{T}^{M G}\left(T^{l}-T_{0}\right)
$$

where $\gamma_{T}^{M G}$ is the rate of change of the surface energy $\gamma^{M G}$ as a function of temperature. $\gamma_{0}^{M G}$ is a reference value of the surface energy evaluated at reference temperature $T_{0}$. An effective pressure is also defined $p^{*}=p+\phi$, which is then substituted into both the bulk and the MG interface equations, transferring $\phi$ to the interface. The value of $T_{\infty}$ is the ambient temperature of the gas phase, also assumed given.

At the CM interface, the unit normal vector pointing from solid into liquid is

$$
\hat{\bar{n}}=\frac{-\bar{h}_{x} \hat{i}+\hat{k}}{\left(1+\bar{h}_{x}^{2}\right)^{1 / 2}},
$$

and the tangential vector is

$$
\hat{\bar{\tau}}=\frac{\hat{i}+\bar{h}_{x} \hat{k}}{\left(1+\bar{h}_{x}^{2}\right)^{1 / 2}} .
$$

The local normal growth speed of the interface is defined as

$$
\bar{v}_{n}=\frac{\bar{h}_{t}}{\left(1+\bar{h}_{x}^{2}\right)^{1 / 2}},
$$

which when positive means growth into the liquid.

The conditions at the CM interface $z=\bar{h}(x, t)$ include the no slip condition for the fluid velocity

$$
\vec{u} \cdot \hat{\bar{\tau}}=0,
$$

where $\vec{u}=(u, w)$ is the fluid velocity vector. Since the CM interface can deform by freezing or melting, there are other interfacial conditions that differ from the conditions applied at 
the surface of a non-deformable solid substrate. First, the temperature at the interface $\left(T_{I}\right)$ is governed by the Gibbs-Thomson equation [21],

$$
T_{I}=T_{M}-\frac{T_{M} \gamma^{C M}}{L_{v}} \bar{K}
$$

Eqn. (0.16) is the condition for local equilibrium at the crystal-melt interface and is appropriate for metals. $T_{M}$ is the melting point of the pure bulk liquid, $L_{v}$ is the latent heat of fusion per unit volume of solid phase, $\gamma^{C M}$ is the (isotropic) CM surface energy and $\bar{K}$ is the curvature of the CM interface which is positive for a crystal sphere.

The difference in conductive heat fluxes through the interface is balanced by the rate of latent heat evolved per unit time and per unit area of interface during growth:

$$
L_{v} \bar{v}_{n}=-k^{l} \nabla T^{l} \cdot \hat{\bar{n}}+k^{s} \nabla T^{s} \cdot \hat{\bar{n}} .
$$

There is also the fluid penetration condition

$$
\frac{\rho^{l}-\rho^{s}}{\rho^{l}} \bar{v}_{n}=\vec{u} \cdot \hat{\bar{n}}
$$

indicating that there is a normal component of the fluid velocity at the CM interface towards (away from) the interface if there is solidification shrinkage, e.g. Al (expansion, e.g. ice).

Finally, a far-field condition is required in order to fix the heat flow out of the film into the crystal. In this work the ' $z$ ' component of the thermal gradient is fixed at a distance $z=-\bar{h}_{c}$ away from the interface, i.e. $\nabla T^{s} \cdot \hat{k}=G_{L}^{a p p}$. The applied thermal gradient in the crystal $G_{L}^{a p p}$ and the ambient temperature in the gas phase $T_{\infty}$ are controllable parameters. It is noted that this particular far-field boundary condition would still be applicable even without assuming a thin solid phase.

\section{The Evolution Equations}

Let $h_{0}$ be the average or initial film thickness in the transverse direction, and $L_{0}$ be a lateral length, which may be defined in terms of a perturbation wavenumber $\omega$ as $L_{0}=2 \pi / \omega$, or, in metallic foams, as the lamellae half-length. Whatever the choice, $h_{0}<<L_{0}$, so that lateral interface shape variations (in the $x$ direction) are much longer than transverse variations (in the $z$ direction). Let the characteristic flow velocity $U_{0}=\nu / L_{0}$. The following variables,

$$
\begin{gathered}
Z=\frac{z}{h_{0}}, \quad X=\frac{x}{L_{0}}, \quad H=\frac{h}{h_{0}}, \bar{H}=\frac{\bar{h}}{h_{0}}, \tau=\frac{U_{0}}{L_{0}} t, \quad U=\frac{u}{U_{0}}, \quad W=\frac{w}{\epsilon U_{0}}, \\
\Phi=\frac{\epsilon^{2} L_{0}}{\mu U_{0}} \phi, \quad P=\frac{\epsilon^{2} L_{0}}{\mu U_{0}} p^{*}, \quad \Theta^{l}=\frac{T^{l}-T_{m}}{T_{m}} \text { and } \Theta^{\mathrm{s}}=\frac{\mathrm{T}^{\mathrm{s}}-\mathrm{T}_{\mathrm{m}}}{\mathrm{T}_{\mathrm{m}}},
\end{gathered}
$$


are used to transform the dimensional equations. In the liquid, the transformed equations of motion are

$$
\begin{gathered}
\epsilon^{2} \operatorname{Re}\left(U_{\tau}+U U_{X}+W U_{Z}\right)=-P_{X}+U_{Z Z}+\epsilon^{2} U_{X X} \\
\epsilon^{4} \operatorname{Re}\left(W_{\tau}+U W_{X}+W W_{Z}\right)=-P_{Z}+\epsilon^{4} W_{X X}+\epsilon^{2} W_{Z Z} .
\end{gathered}
$$

where $R e$ is the Reynolds number $R e=U_{0} L_{0} / \nu$, and the kinematic viscosity $\nu=\mu / \rho^{l}$. The continuity condition is

$$
U_{X}+W_{Z}=0 .
$$

Temperature in the bulk liquid and solid is governed by

$$
\epsilon^{2} \operatorname{Pr} \operatorname{Re}\left(\Theta_{\tau}^{l}+U \Theta_{X}^{l}+W \Theta_{Z}^{l}\right)=\Theta_{Z Z}^{l}+\epsilon^{2} \Theta_{X X}^{l}
$$

and

$$
\epsilon^{2} \operatorname{Pr} \operatorname{Re} \Theta_{\tau}^{s}=\Theta_{Z Z}^{s}+\epsilon^{2} \Theta_{X X}^{s}
$$

respectively. The Prandtl number $\operatorname{Pr}=\nu / \alpha$, is the ratio of the kinematic viscosity to the thermal diffusivity $\alpha_{L}$.

At the MG interface,

$$
\begin{gathered}
-P+P_{G}+\epsilon^{2} \Phi+2 \epsilon^{2}\left[W_{Z}+\epsilon^{2} U_{X} H_{X}^{2}-H_{X} U_{Z}-\epsilon^{2} H_{X} W_{X}\right]\left(1+\epsilon^{2} H_{X}^{2}\right)^{-1 / 2} \\
=\bar{C}^{-1}\left[1-M_{T}\left(\Theta^{l}-\Theta_{\gamma}^{l}\right)\right] H_{X X}\left(1+\epsilon^{2} H_{X}^{2}\right)^{-3 / 2} \\
\left(U_{Z}+\epsilon^{2} W_{X}\right)\left(1-\epsilon^{2} H_{X}^{2}\right)-2 \epsilon^{2}\left[U_{X}-W_{Z}\right] H_{X} \\
=-\bar{M}_{T}\left[\Theta_{X}^{l}+H_{X} \Theta_{Z}^{l}\right]\left(1+\epsilon^{2} H_{X}^{2}\right)^{1 / 2} \\
W=H_{\tau}+U H_{X}
\end{gathered}
$$

and

$$
\Theta_{Z}^{l}+\epsilon^{2} \Theta_{X}^{l} H_{X}+B i\left[\Theta^{l}-\Theta_{\infty}\right]\left(1+\epsilon^{2} H_{X}^{2}\right)^{1 / 2}=0 .
$$

The Biot number is $B i=\alpha_{T h} h_{0} / k^{l}$. The scaled temperature in the gas far from the MG interface $\Theta_{\infty}=\left(T_{\infty}-T_{m}\right) / T_{m}$. The modified inverse capillary number $\bar{C}^{-1}=\epsilon^{3} C^{-1}$, and modified Marangoni number, $\bar{M}_{T}=\epsilon M_{T} C^{-1}$, have been scaled in order to retain the influence of MG interfacial effects at leading order.

In this analysis, the body force potential $\Phi(X, T)$ includes van der Waals dispersion forces which drive the instability of the layer. The gradient of the potential is [22]

$$
\Phi_{X}=\frac{-3 \tilde{A}(H-\bar{H})_{X}}{(H-\bar{H})^{4}}
$$

where $\tilde{A}=A^{\prime} /\left(6 \pi h_{0} \rho^{l} \nu^{2}\right)$ is a non-dimensional Hamaker constant.

The boundary conditions at the CM interface transform to

$$
\Theta_{I}=\overline{\Gamma H}_{X X}\left[1+\epsilon^{2} \bar{H}_{X}^{2}\right]^{-3 / 2}
$$




$$
\begin{gathered}
W=(1-E) \bar{H}_{\tau}+\bar{H}_{X} U, \\
U+\epsilon^{2} \bar{H}_{X} W=0
\end{gathered}
$$

and

$$
\hat{\hat{L}} \bar{H}_{\tau}=\beta_{T} \Theta_{Z}^{s}-\Theta_{Z}^{l}+\epsilon^{2}\left[\Theta_{X}^{l}-\beta_{T} \Theta_{X}^{s}\right] \bar{H}_{X} .
$$

The non-dimensional latent heat of fusion is $\hat{\hat{L}}=\epsilon L_{v} h_{0} U_{0} /\left(T_{m} k^{l}\right)$, the capillary coefficient is $\Gamma=\gamma^{C M} /\left(L_{V} h_{0}\right)$ and $E$ is the ratio of the solid to liquid density. The CM surface energy has been retained by introducing $\bar{\Gamma} \equiv \epsilon^{2} \Gamma$.

At $Z=-\bar{H}_{c}$ the vertical component of the temperature gradient is fixed as

$$
\Theta_{Z}^{s}=G_{S},
$$

where $G_{S}=G_{L}^{a p p} h_{0} / T_{m}$ in scaled variables.

In order to compare with Williams and Davis [16] and with results of [1], the independent variables are rescaled as $\hat{\tau}=\left[3 \tilde{A}^{2} / \bar{C}^{-1}\right] \tau$ and $\hat{X}=\left[\sqrt{3 \tilde{A} / \bar{C}^{-1}}\right] X$, based on MG capillary and van der Waals forces. Marangoni effects are assumed to be negligible in order to focus on the effect of the crystal-melt surface energy. (In [1], the value of the Marangoni coefficient was erroneously underestimated. Nevertheless, it is stated that whether or not thermal Marangoni effects are important will depend on the system under consideration. These effects will be the focus of future work.) The resulting evolution equations governing the $\mathrm{CM}$ and the MG interfaces become:

$$
\bar{H}_{\hat{\tau}}=B i^{2} R\left[\frac{H-\bar{H}-1}{(1+B i)(1+B i(H-\bar{H}))}\right]+\frac{B i \mathcal{G} \bar{H}_{\hat{X} \hat{X}}}{1+B i(H-\bar{H})}
$$

and

$$
H_{\hat{\tau}}-(1-E) \bar{H}_{\hat{\tau}}=-\left[\frac{(H-\bar{H})_{\hat{X}}}{(H-\bar{H})}+(H-\bar{H})^{3} H_{\hat{X} \hat{X} \hat{X}}\right]_{\hat{X}}
$$

Four parameters are present in (0.36) and (0.37): $E$, the ratio of the solid to liquid densities, the Biot number $B i$, the thermal/solidification parameter $R=\bar{C}^{-1} \Theta_{\infty} /\left(3 \tilde{A}^{2} \hat{L}\right)$, and $\mathcal{G}=$ $\bar{\Gamma} / \hat{\hat{L}} \tilde{A}$, a new CM surface energy parameter. In Eqn. (0.36), the heat flux through the solid has been chosen so that a uniform layer of liquid remains static. To maintain such a time independent base state, the heat flux through the crystal must balance the heat flux in the liquid at the $\mathrm{CM}$ interface, so that

$$
\beta_{T} \Theta_{Z}^{S}=R \frac{B i}{(1+B i)} .
$$

The static base state allows the interface perturbations to be represented by normal modes. The pair of equations (0.36) and (0.37) that govern morphological evolution of the CM and MG interfaces include the CM interface energy. 


\section{Linear Stability}

In order to provide a representative benchmark for comparison to previous analyses, we examine the stability of the static, uniform melt layer. The governing equations are expanded and the base state is governed by $\bar{H}_{\hat{\tau}}^{(0)}=H_{\hat{\tau}}^{(0)}=0$, with $H^{(0)}=1$ and $\bar{H}^{(0)}=0$. (The superscripts ${ }^{(0)}$ refer to base states and ${ }^{(1)}$ refer to perturbed states.) The linearized perturbation equations are:

$$
\bar{H}_{\hat{\tau}}^{(1)}=\frac{R B i^{2}}{(1+B i)^{2}}\left(H^{(1)}-\bar{H}^{(1)}\right)+\frac{B i \mathcal{G}}{(1+B i)} \bar{H}_{\hat{X} \hat{X}}^{(1)}
$$

and

$$
H_{\hat{\tau}}^{(1)}-(1-E) \bar{H}_{\hat{\tau}}^{(1)}=-\left(H^{(1)}-\bar{H}^{(1)}\right)_{\hat{X} \hat{X}}-H_{\hat{X} \hat{X} \hat{X} \hat{X}}^{(1)} .
$$

The shape perturbations are written as normal modes,

$$
H^{(1)}=\rho \exp (\sigma \hat{\tau}) \exp (i \omega \hat{X})
$$

and

$$
\bar{H}^{(1)}=\bar{\rho} \exp (\sigma \hat{\tau}) \exp (i \omega \hat{X}),
$$

where $\sigma$ is the growth rate and $\omega$ is the wavenumber of the perturbation. Substituting the perturbed shapes into (0.39) and (0.40) leads to the dispersion relation,

$$
\sigma^{2}+\left(B+(G-1) \omega^{2}+\omega^{4}\right) \sigma+(B-G) \omega^{4}+G \omega^{6}=0
$$

that depends on the two parameters $B=\bar{C}^{-1} B i^{2} \Theta_{\infty} /\left(3 \hat{\hat{L}} \tilde{A}^{2}(1+B i)^{2}\right)$ and $G=\mathcal{G} B i /(1+B i)$. There is the condition relating the perturbation amplitudes:

$$
\frac{\bar{\rho}}{\rho}=\frac{B}{\sigma+G \omega^{2}+B}
$$

which fixes the relative magnitudes and the relative lateral positions of the $\mathrm{CM}$ and $\mathrm{MG}$ interface perturbations.

The case with zero surface energy $(G=0)$ is presented in [1] and is briefly reviewed here. The marginal stability condition (vanishing of the real part of $\sigma$ ) is given by the heavy black curve presented in Fig. 2, on a diagram of $B$ vs. $\omega$. (In Fig. 2, $E=1$ ). Within the marginal curve, perturbations are unstable, outside the curve they are stable. The benchmark results of Williams and Davis [16], corresponding to an isothermal, flat-CM interface case, occur at $B=0$. A range of unstable wavenumbers is exhibited. However, as $B$ is increased from 0 , the range of unstable perturbation wavenumbers shrinks culminating in a maximum value of $B_{C}=0.25$ along the marginal stability surface. As illustrated in the figure, for $B \neq 0$, all marginally stable states are oscillatory.

As discussed previously [1], compared to the isothermal case, the capillary and the van der Waals forces couple with the thermal/solidification effects to produce oscillatory instability. 


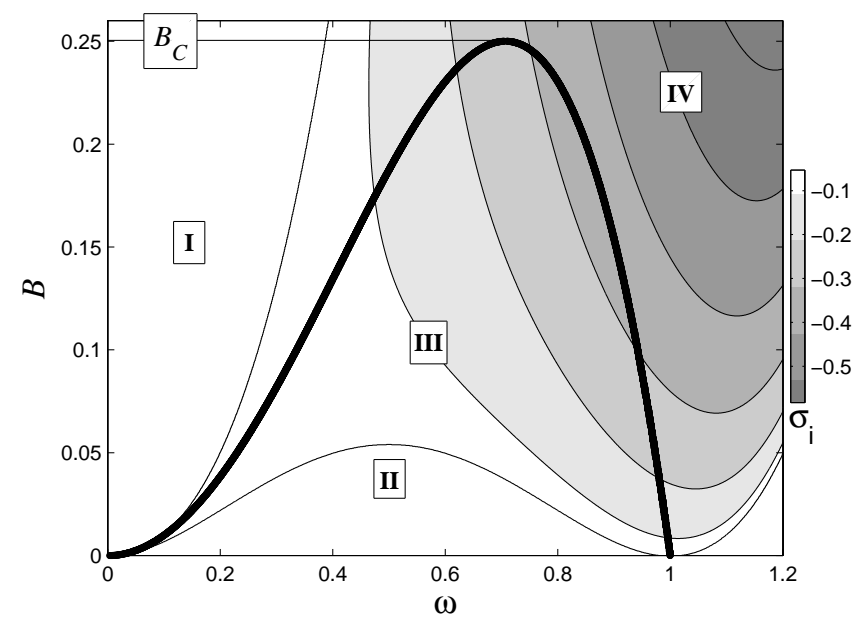

Figure 2: Stability plot as a function of the thermal parameter $B$ in the absence of CM interface energy with $E=1$ and no Marangoni effects. The heavy black curve is the marginal stability limit. Perturbation conditions below this curve are unstable, and conditions above are stable. Onset of instability occurs at a critical thermal parameter $B_{C}=0.25$. The white regions represent stationary initial conditions, while the grey-scale contours represent iso-frequency oscillatory states. Region I is therefore stationary and stable. Region II is stationary but unstable, region III, is oscillatory and unstable, and region IV is oscillatory and stable. (Taken from [1]). 
The fact that the crystal-melt interface is deformable impacts the relative importance of the thermal, capillary and van der Waals effects. The applied thermal gradient acts in direct opposition to the van der Waals forces at the narrowest region of the film and, therefore, the film melts back in the region as the rupture tip approaches. Small amplitude initial symmetric perturbations grow to rupture as standing waves and with increasing number of oscillations the closer the initial configuration is to the marginal stability surface. Moreover, for larger initial shape perturbations the region of instability on a plot of the thermal parameter $B$ versus the wavenumber $\omega$ increases and the time to rupture decreases; these are nonlinear effects.

Turning now to the case with crystal-melt surface energy $(G \neq 0$ and $E=1)$, an example of the dispersion relation is given in Fig. (3). There are two unstable regions where $\sigma_{\text {real }}>0$. In the range $0.45<\omega<0.78$ there is an oscillatory shorter-wave instability driven by van der Waals effects, as seen in the previous cases without CM surface energy. The other region is $0<\omega<0.45$, which is a stationary instability, since $\sigma_{\text {imag }}=0$ in this range. This latter, new 'longer-wave' instability arises as a consequence of the nonisothermal CM interface (i.e. a CM interface which is now truly at local equilibrium).
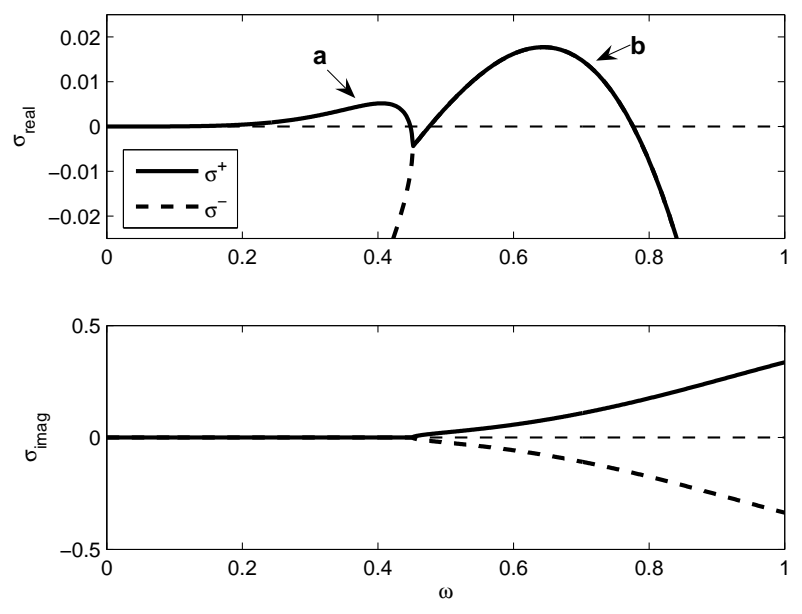

Figure 3: Dispersion relation with CM surface energy. Crystal-melt surface energy introduces a new long-wave instability for $\omega<0.45$ in this example with $B=0.14$ and $G=0.17$. The upper curves are the real components of the growth rate, and the lower curves are the imaginary components. The positive real growth rates in the range $0<\omega<0.45$ correspond to the long-wave instability and are labeled $\mathbf{a}$, while the shorter wavelength van der Waals attractive instability mode is labeled $\mathbf{b}$.

The effect of $G$ on marginal stability is apparent when comparing Fig. (4) to Fig. (2). In Fig. (4), $G=0.10$, which is based on dimensional values of the material properties for pure aluminum [23] [24]. A local maximum of the marginal stability surface at $\omega=0$ and $B_{C 2}=0.10$ arises due to the new long-wave mode of instability. The critical value $B=B_{C 1}$ at 
the onset of instability is reduced from 0.25 to 0.2 due to CM surface energy. Another effect of the CM surface energy is apparent when comparing the marginal stability surface in Fig. (4) with Fig. (2). Previously, the region of oscillatory instability (region III) stretched in a ever-narrowing band of wavenumbers, terminating at the origin. The presence of CM surface energy effectively clips the lower wavenumber oscillatory linearly unstable region in Fig. (4), at $\omega=0.34$. However, even though wavenumbers below this limit have real growth rates, we will see that disturbances of low wavenumber can excite higher wavenumber oscillatory modes.

The new longer-wave unstable modes are always neutrally stable $\left(\sigma_{\text {real }}=0\right)$ at $\omega=0$, and are stationary in time. As the control parameter $B$ is reduced to the point $B_{C 2}$, the local maximum of the marginal stability curve is at vanishing wavenumber. However, as $B$ is reduced below $B_{C 2}$, the fastest growing mode is finite and increases as $B$ is reduced further. Marginally stable or unstable modes at vanishing wavenumber have been investigated by Goldstone et. al. [25] and Magyari et. al. [26] and have been observed to manifest as zero frequency translation solitons [27] and in electrohydrodynamic homeotropic nematic layers [28]. Nonlinear interaction between an unstable mode and a slow but stable Goldstone mode has been studied in a system of coupled Burgers equations [29].

The physical mechanism driving the appearance of the expanded region of linear instability with surface energy is a manifestation of the coupling between the interfaces. In classical directional solidification and during free crystal growth, solid-liquid surface energy acts to stabilize the crystal-melt interface. This can be understood by considering the GibbsThompson equation for an isotropic, pure material,

$$
T_{I}=T_{M}-\frac{T_{M} \gamma^{C M}}{L_{v}} \bar{K}
$$

According to this equation, a convex region on the crystal interface that curves upward into the melt will have a lower melting temperature. Hence, if the melt is hotter, then it is easier for the convex region to melt back into the crystal. Conversely, a concave region on the interface will have an increased melting temperature, creating an impediment to further melting. This stabilizing effect is also present in the two interface coupled system, and influences the stability diagrams as will be discussed below. Nevertheless, for a sufficiently large value of $G$, the unstable longer-wave modes persist.

We can examine this case by separating the real and imaginary components of the dispersion relationship to give:

$$
\sigma_{r}^{2}-\sigma_{i}^{2}+\left(B+(G-1) \omega^{2}+\omega^{4}\right) \sigma_{r}+(B-G) \omega^{4}+G \omega^{6}=0
$$

and

$$
\sigma_{i}\left(2 \sigma_{r}+\left(B+(G-1) \omega^{2}+\omega^{4}\right)\right)=0 .
$$

$G$ is a positive real parameter, and for $0<G<1$, the term $(G-1) \omega^{2}$ represents competition between destabilizing van der Waals attraction and stabilizing CM surface energy. For $G$ small enough, $(G-1) \omega^{2}$ is negative and contributes to a positive growth rate. But as $G$ 
increases $(G-1) \omega^{2}$ becomes less negative, thus promoting stability as the CM surface energy overrides the van der Waals attraction.

The term in the dispersion relation $(B-G) \omega^{4}$ is a term that would not be present for an isolated CM interface. So, with two interfaces, not only does the crystal-melt surface energy compete against the van der Waals effects, but it also modifies the otherwise stabilizing thermal effects. If $G>B$ then the term $(B-G) \omega^{4}$ is negative, resulting in a positive contribution to the growth rate for lowest wavenumber perturbations.

In addition, since the imaginary component of the growth rate is zero within the range of unstable perturbation wavenumbers (for instance, below $\omega=0.45$ as shown in Fig. 3), therefore (0.46) may be written

$$
\left(\sigma_{r}+B+G \omega^{2}\right)\left(\sigma_{r}-\omega^{2}+\omega^{4}\right)+\omega^{2} B=0,
$$

and if $\sigma_{r}<<G \omega^{2}$ and $B<<G \omega^{2}$ then

$$
\sigma_{r} \sim \omega^{2}-\omega^{4}-\frac{B}{G}
$$

Furthermore, from (0.49) a critical wavenumber for the nonoscillatory branch can be found by setting $\sigma_{r}=0$ giving

$$
\omega_{c}=\sqrt{1-\frac{B}{G}}
$$

The ratio of $B / G$ then is a parameter which governs the cutoff wavenumber for the unstable, non-oscillatory modes[30].

Surface energy properties near the melting point vary from material to material. Aluminum has a surface energy ratio $\gamma_{0}^{C M} / \gamma_{0}^{M G}=0.10$ [23] [24]. Lead has a slightly higher ratio of 0.12 [5], while succinonitrile (a material of significant experimental interest as a model for metals and alloys) has a ratio of 0.23 [31]. Hence, by holding all other properties constant but varying the crystal-melt surface energy, a means is provided for investigating the stability of different material systems, with varying crystal-melt to melt-gas surface energy ratios. Figs. (4) to (6) are a series of plots that shows how CM surface energy affects the marginal stability condition. As crystal-melt surface energy is increased there is increased moderation of the linear behavior observed in the absence of crystal-melt surface energy. As $G$ is increased to a value of 0.17 in Fig. (5) the two maxima of the marginal stability surface have the same values of $B$, i.e. $B_{C 1}=B_{C 2}=0.17$. For values of $B$ just below the double maximum, however, the shorter-wave instabilities near $\omega=0.64$ have much larger growth rates than the longer-wave modes. As the CM surface energy parameter is further increased, the parameter range over which the stationary long-wave modes of instability are observed grows even larger at the expense of the oscillatory shorter-wave instability as seen in Fig. (6). Now the prominent "lobe" of oscillatory instability exhibited in Figs. (4) and (5) is no longer apparent. 


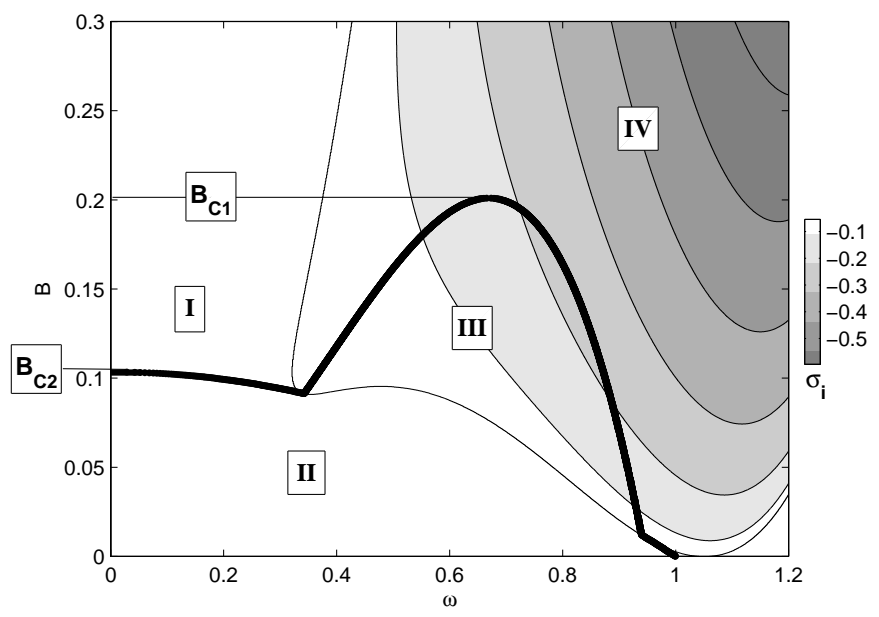

Figure 4: Stability diagram with $G=0.10$. Marginal stability surface is given by the heavy curve. As in the previous stability diagrams, region I is stable and stationary, region II is unstable and stationary, region III is unstable and oscillatory and region IV is stable and oscillatory. The gray-scale corresponds to the magnitude of the imaginary component of the growth rate. The critical thermal parameter $B_{C 1}=0.20$ corresponds to the onset of the van der Waals instability mode. The second critical point is for the onset of the $(\omega=0)$ instability mode at $B_{C 2}=G=0.10$.

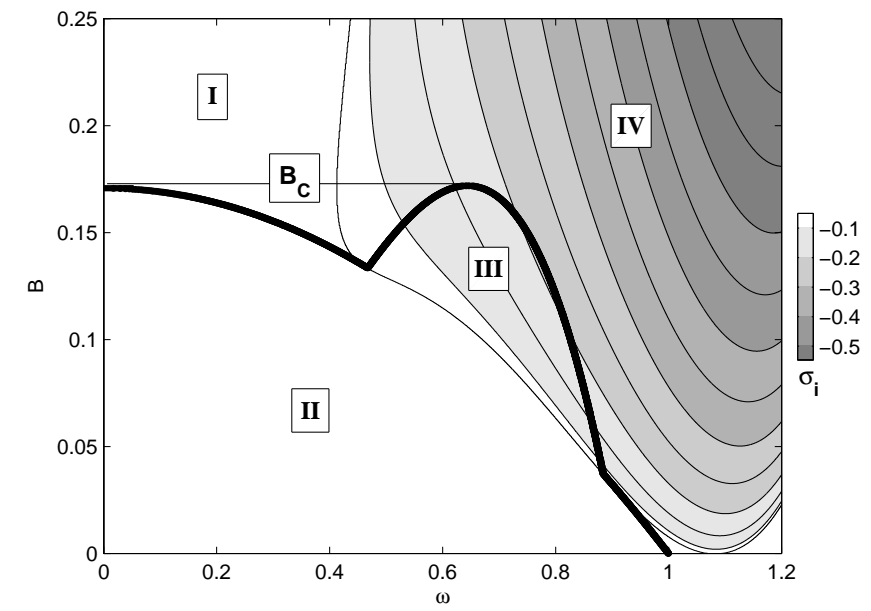

Figure 5: Stability diagram with $G=0.17$. Marginal stability surface at the "double critical" value of the CM surface energy parameter $G=0.17$. The regions I - IV and the gray-scale are as defined in Fig. (4). The critical value $B_{C}=G=0.17$. 


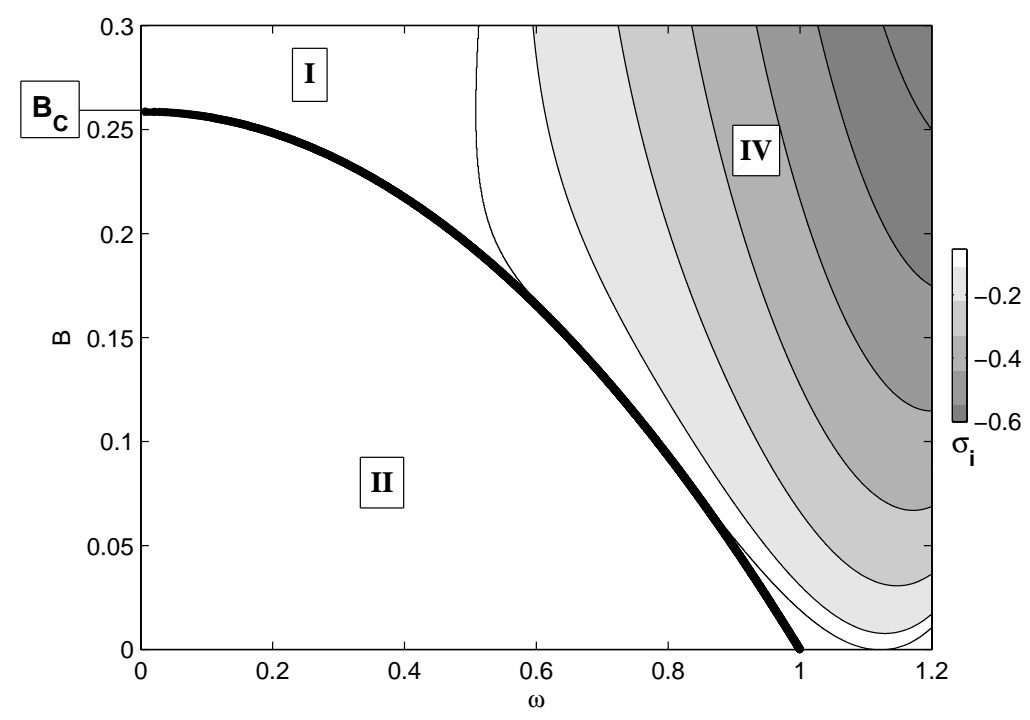

Figure 6: Stability diagram with $G=0.26$. Marginal stability surface for a higher value of the CM surface energy parameter $G=B_{C}=0.26$. The regions I, II and IV and the gray-scale are as defined in Fig. (4). Note that there is no longer an unstable oscillatory region.

Fig. (7) plots results for four values of the CM surface energy parameter $G$. In summary, as $G$ increases, the longer-wave stationary mode becomes ever more important and the range of unstable values of $B$ increases with increasing $G$.

\section{Interface Evolution with Crystal-melt Surface Energy}

A modified, spatially second-order accurate conservative method is used to discretize Eqns. (0.36) and (0.37) and the results are updated in time using the MATLAB function ode15s which employs backwards time differencing $[32,33]$. Periodic boundary conditions are applied at the ends of the domain for both interfaces, and it was found that this differencing method is stable. (More details are provided in [1]). For all calculations, the length of the computational domain is equal to the length of the initial perturbation wavelength.

Fig. 8 compares rupture times predicted by linear theory (solid curves) computed using $\sigma_{r}$, to the numerically calculated times (dots) for the "double critical" surface tension parameter value $G=0.17$. The initial perturbations are given by equations (0.41) and $(0.42)$ with $\rho=0.01$, and are aligned to grow as standing waves. The rupture time data for Fig. (8) was gathered for each wavenumber using $N=200$ and $N=400$ spatial nodes to dis- 


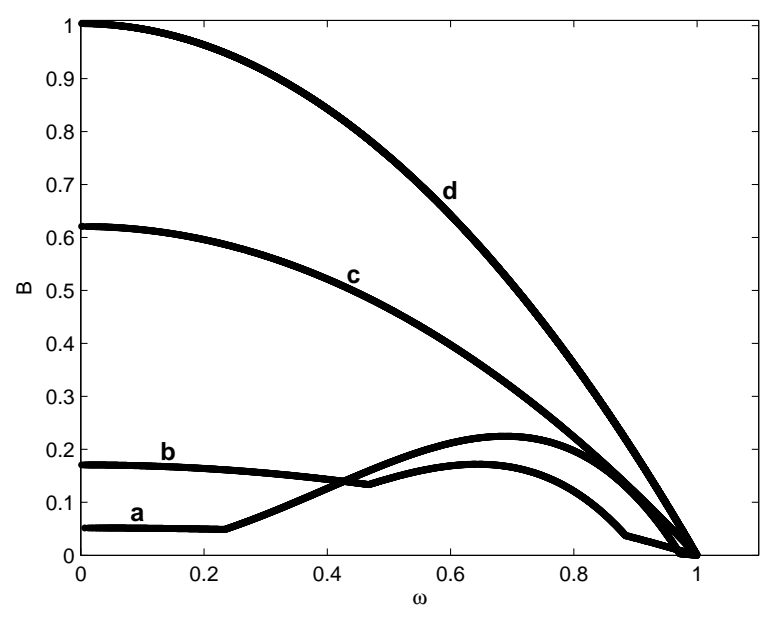

Figure 7: Composite of marginal stability surfaces with CM surface energy. The curves a-d are for $G=0.05, G=0.17, G=0.62, G=1.00$, respectively. The curves separate unstable perturbation conditions below from stable conditions above.

cretize both the MG and CM interfaces. Convergence of the results was observed for all calculations above $\omega \sim 0.15$. At lower wavenumbers, not all of the interface shape solutions and rupture times converged with increasing $N$. The divergence may be due to limitations in the numerical technique, or it may be due to the onset of chaotic behavior. In general, non-linear effects tend to speed up rupture times for initial perturbations with wave numbers in the range $0.37<\omega<0.78$. Below $\omega=0.37$, however, the numerical solutions show additional complexity. This is due to interaction between disturbances of different wavelength. Recall that, in this figure the left lobe range of wavenumbers $0<\omega<0.46$ corresponds to the long-wave stationary instability, while the right lobe range of wavenumbers between $0.48<\omega<0.78$ corresponds to a short-wave, oscillatory instability.

Since the lateral end conditions used in the numerical model are periodic, only integer multiples of the initial perturbation wavenumber can be excited by nonlinearity. As the wavenumber drops below $\omega=0.37$, the first harmonic of the perturbation is also (barely) linearly unstable, and has a much higher growth rate. Therefore, as this mode is excited by nonlinearity at still lower wavenumbers, the rupture time decreases. Also notice that there is a peak in the numerical time to rupture at $\omega=0.23$, because the next higher integer multiple, $2 \omega=0.46$, is at the start of the stable band $0.46<\omega<0.48$, so that it is not excited and, therefore, growth is dominated by the slower-growing fundamental mode only. Another feature of Fig. (8) are the step-ups in the time to rupture between $\omega=0.63$ and $\omega=0.64$ and between $\omega=0.68$ and $\omega=0.69$ (other instances are not clearly visible on the plot.) This is due to half-integer increases in the number of oscillations of the growing perturbation before rupture.

Morphological evolution of the films at several times are shown in figure 9, corresponding 


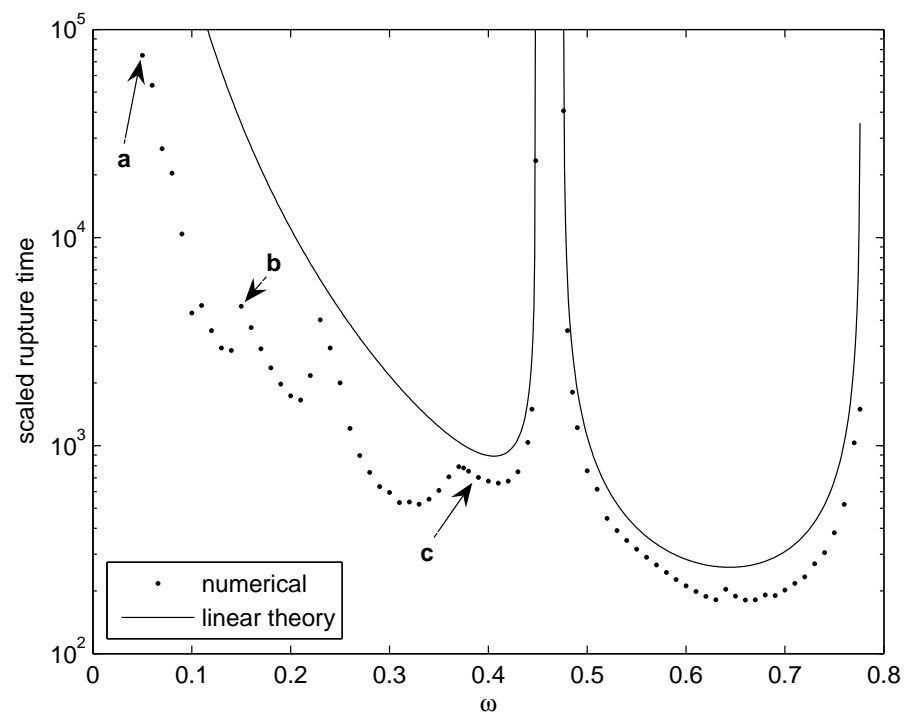

Figure 8: Rupture time varies with $\omega$ for surface tension parameter $G=0.17$. Amplitude of the initial perturbation is 0.01 and $N=200$ for all cases. For all calculations, the length of the computational domain is equal to the length of the initial perturbation wavelength. The curves give the rupture time prediction from linear theory, and the dots are numerically calculated rupture times. Interface shape evolution for the points labeled $\mathbf{a}, \mathbf{b}$ and $\mathbf{c}$ are plotted in figure 9 . 
to the points labeled $\mathbf{a}, \mathbf{b}$ and $\mathbf{c}$ in figure 8 . The initial perturbation in figure $9 \mathbf{a}$ has wavenumber $\omega=0.05$, but the mode that ruptures is $\omega=0.6$, or approximately 12 times the initial perturbation. Since $\omega=0.6$ has a much higher growth rate, once excited it grows more rapidly than the initial perturbation. In Fig. (9b) the initial perturbation is $\omega=0.15$ and a higher mode is excited $(\omega=2 \times 0.15=0.30)$ leading to stationary growth and rupture. Since higher modes also have higher growth rates, it is reasonable that the time to rupture is much shorter than predicted by linear theory. Fig. (9c) is the evolution for an initial perturbation of $\omega=0.39$. Growth is non-oscillatory and higher modes are not being excited. In this case, the numerical time to rupture exhibits the nonlinear effects characteristic of a single linearly unstable mode (as shown for example in the benchmark studies [1] and Williams and Davis [16].)

\section{Conclusions}

The effect of the crystal-melt (CM) surface energy on instability and evolution of a thin melt film in a temperature gradient is considered. A governing pair of non-linear thin-film equations governs the crystal-melt and melt-gas interfaces bounding a melt film. As in the case without CM surface energy, one practical result of this work is that there is a maximum applied far field temperature in the inert gas, beyond which there is no instability. The effect of the CM surface energy is to expand the range of linearly unstable perturbations in the parameter space $B=\bar{C}^{-1} B i^{2} \Theta_{\infty} /\left(3 \hat{\hat{L}} \tilde{A}^{2}(1+B i)^{2}\right)$ (the thermal solidification parameter) vs. $\omega$ (the perturbation wavenumber). The new region of instability is longer-wave and is stationary, compared to the instabilities observed in the absence of the crystal-melt surface energy which are only shorter wave-length and oscillatory, (the latter of which are governed by van der Waals, MG capillary and thermal effects). The new instabilities arise due to the effect of the Gibbs-Thomson condition on the coupling between the MG and CM interfaces through the thermal field. By tuning the ratio of crystal-melt to gas-melt interfacial energies, the maxima in the marginal stability surface can be made to coincide, a multiple bifurcation point.

Numerical analysis reveals dynamics of the two interfaces up to a time just before rupture. Initial conditions were configured so that growth is in the form of standing waves. Previous work reveals that oscillations occur by competition between stabilizing thermal effects and destabilizing van der Waals effects. The rise in heat flux in thinning regions of the film drives melt-back of the CM interface. With CM surface energy the numerical solutions show interactions between the two modes of instability. Initially longer (stationary) wavelength instabilities have very slow growth rates and can lead to the activation of shorter (oscillatory) instabilities that grow much more rapidly, eventually leading to rupture by means of the faster growing modes.

Finally, we comment that the emergence of a long wave instability due to the crystal-melt surface energy has mathematical features similar to the emergence of a long-wavelength in- 

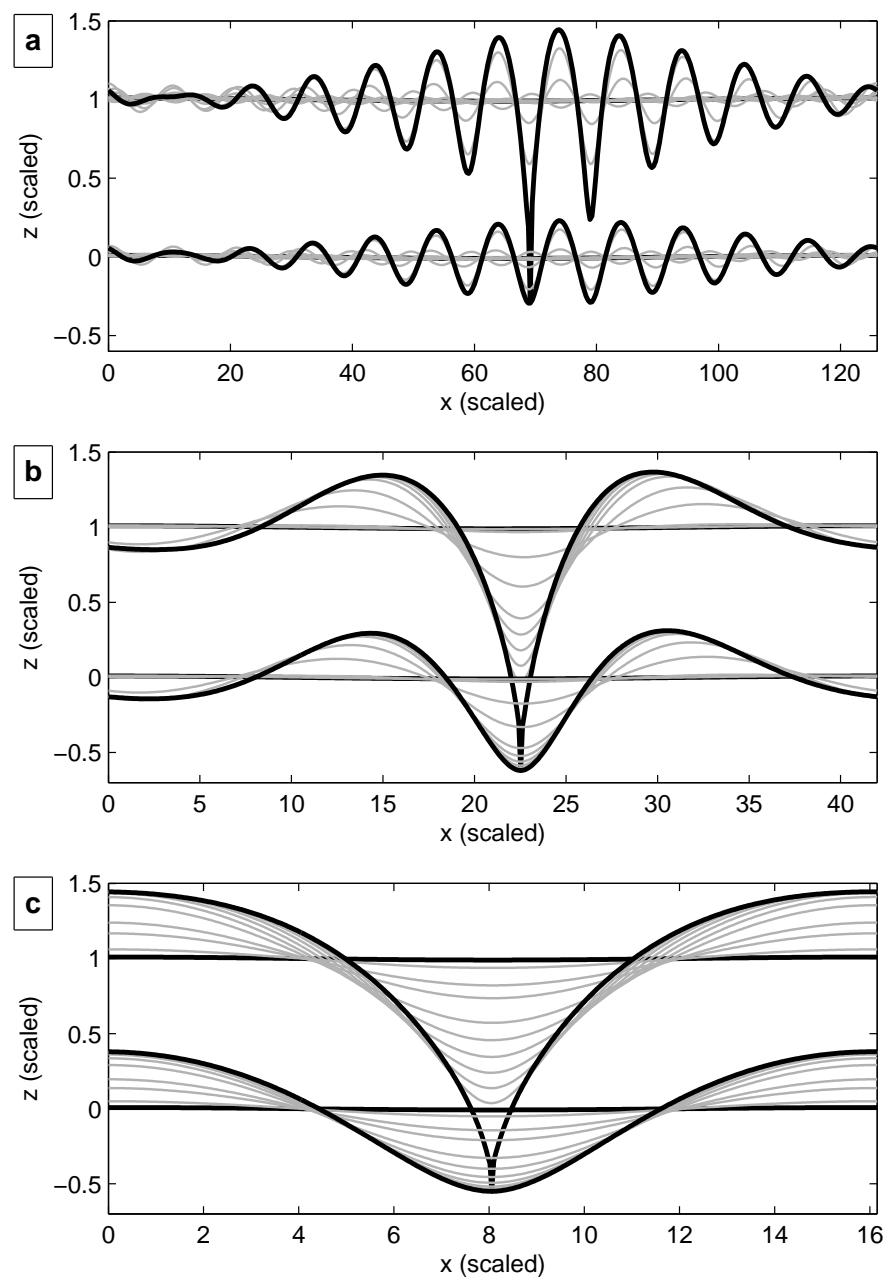

Figure 9: Evolution of both interfaces at wavenumbers that correspond to the labeled points in figure 8. Plot $\mathbf{a}$ is for $\omega=0.05, \mathbf{b}$ is for $\omega=0.15$ and $\mathbf{c}$ is for $\omega=0.39$. The heavy dark curves are the initial and final interface shapes, and the light curves are at intermediate times. For all calculations, the length of the computational domain is equal to the length of the initial perturbation wavelength. 
stability and dry-patch formation in surface-energy-driven Benard convection [34], although the physical processes are not related.

\section{Acknowledgements}

The authors acknowledge helpful discussions with S.H. Davis and A.A. Golovin.

\section{References}

[1] M. Beerman, L. N. Brush. Oscillatory instability and rupture in a thin melt film on its crystal subject to melting and freezing. J. Fluid Mech., 586 (2007), 423-448.

[2] J. G. Dash, H. Fu, J.S. Wettlaufer, The premelting of ice and its environmental consequences. Rep. Prog. Phys., 58 (1995), 115-167.

[3] U. Tartaglino, T. Zykova-Timan, F. Ercolessi, E. Tosatti. Melting and nonmelting of solid surfaces and nanosytems. Phys. Reports, 411 (2005), 291-321.

[4] J. S. Wettlaufer, M. G. Worster. A theory of premelting dynamics. Ann. Rev. Fluid Mech., 76 (2006), 3602-3635.

[5] B. Pluis, A. W. Denier van der Gon, J. W. M. Frenken, J. F. van der Veen. Crystal-face dependence of surface melting. Phys. Rev. Lett., 59 (1987), No. 23, 2678-2681.

[6] M. F. Ashby, A. G. Evans, N. A. Fleck, L. J. Gibson, J. W. Hutchinson, H. N. G. Wadley. Metal Foams: A Design Guide. Butterworth-Heinemann, 2000.

[7] J. Banhart. Manufacture, characterization and application of cellular metals and metal foams. Prog. Mat. Sc., 46 (2001), 559-632.

[8] C. C. Yang,H. Nakae, H. The effects of viscosity and cooling conditions on the formability of aluminum alloy. Jour. Mater. Proc. Tech., 141 (2003), 202-206.

[9] C. J. W. Breward. The Mathematics of Foam. Ph.D. dissertation, Oxford University, 1999.

[10] L. N. Brush, S. H. Davis. A new law of thinning in foam dynamics. J. Fluid Mech., 534 (2005), 227-236.

[11] R. M. Lorenz, C. L. Kuyper, P. B. Allen, L. P. Lee, D. T. Chiu. Direct laser writing on electrolessly deposited thin metal films for applications in micro- and nanofluidics. Langmuir, 20 (2004), 1833-1838.

[12] D. A. Willis, X. Xu. Transport phenomenon and droplet formation during pulsed laser interaction with thin films. J. Heat Trans., 122 (2000), 763-770. 
[13] V. S. Ajaev, V. A. Willis. Heat transfer, phase change, and thermocapillary flow in films of molten metal on a substrate. Num. Heat Trans. Pt. A, 50 (2006), 301-313.

[14] D. A. Willis, X. Xu, C. C. Poon, A. C. Tam. Laser-assisted surface modification of thin chromium films. Opt. Eng., 37 (1998), No. 3, 1033-1041.

[15] A. Oron, S. H. Davis, S. G. Bankoff. Long-scale evolution of thin liquid films. Rev. Mod. Phys., 69 (1997), No. 3, 931-980.

[16] M. B. Williams, S. H. Davis. Non-linear theory of film rupture. J. Coll. Int. Sc., 90 (1982), No. 1, 220-228.

[17] J. P. Burelbach, S. G. Bankoff and S. H. Davis. Nonlinear stability of evaporating/condensing liquid films. J. Fluid Mech., 195 (1988), 463-494.

[18] R. O. Grigoriev. Control of evaporatively driven instabilities of thin liquid films. Phys. Fluids, 14 (2002), No. 6, 1895-1909.

[19] D. Bandyopadhyay, R. Gulabani, A. Sharma. Instability and dynamics of thin liquid bilayers. Ind. Eng. Chem. Res., 44 (2005), 1259-1272.

[20] L. S. Fisher, A. A. Golovin. Nonlinear stability analysis of a two layer thin liquid film: dewetting and autophobic behavior J. Coll. Int. Sci., 291 (2005), 515-528.

[21] A. A. Wheeler. In the handbook of crystal growth, Vol. 1. Elsevier Science Publishers B.V., 1993.

[22] J. N. Israelachvili. Intermolecular and surface forces. Academic Press, London, 1991.

[23] W. Kurz, D. J. Fisher. Fundamentals of Solidification. Trans. Tech. Publications, 1994.

[24] J. P. Anson, R. A. L. Drew, J. E. Gruzleski. The surface tension of molten aluminum and Al-Si-Mg alloy under vacuum and hydrogen atmospheres. Met. Mater. Trans. B, 30 (1999), 1027.

[25] J. Goldstone, R. Jackiw. Quantization of nonlinear waves. Phys. Rev. D, 11 (1975), No. 6, 1486-1498.

[26] E. Magyari, H. Thomas. Defective degeneracy of the solitary-wave Goldstone mode. Phys. Rev. Lett., 53 (1984), No. 20, 1866-1869.

[27] H. C. Fogedby. Solitons and diffusive modes in the noiseless Burgers equation: Stability analysis. Phys. Rev. E, 57 (1998), No. 2, 2331-2337.

[28] A. Hertrich, W. Decker, W. Pesch, L. Kramer. The electrohydrodynamic instability in homeotropic nematic layers. J. Phy. II, 2 (1992), 1915-1930. 
[29] E. A. Glasman, A. A. Golovin, A. A. Nepomnyashchy. Instabilities of wavy patterns governed by coupled Burgers equations. SIAM J. Appl. Math., 65 (2004), No. 1, 230251.

[30] S. H. Davis. personal communication. 2007, Northwestern University, Department of Engineering Science and Applied Mathematics.

[31] L. M. Williams, M. Muschol, X. Qian, W. Losert, H. Z. Cummins. Dendritic sidebranching with periodic localized perturbations: Directional solidification of pivalic acidcoumarin 152 mixtures. Phys. Rev. E, 48 (1993), No. 1, 489-500.

[32] L. F. Shampine, M. W. Reichelt, The MATLAB ode suite. SIAM J. Scien. Comp., 18 (1997), No. 1, 1-22.

[33] C. F. Gerald, P. O. Wheatley. Applied Numerical Analysis. Addison - Wesley Publishing Company, Inc., 1984.

[34] S. J. Vanhook, M. F. Schatz, W. D. McCormick, J. B. Swift, H. L. Swinney. LongWavelength Instability in Surface-Tension-Driven Benard Convection. Phys. Rev. Lett., 75 (1995), No. 24, 4397-4400. 